\title{
Einsatz von Künstlicher Intelligenz (KI) für die Optimierung von Pla- nungsprozessen im Wasserbau
}

\author{
Patrycja-Jadwiga Sankowska \\ Nina Kumbruck \\ Dr. Christian Leyh \\ Dr. Andreas Niekler \\ Dr. Daniel Wiegreffe
}

\begin{abstract}
Planungs- und Bauvorhaben werden durch zunehmende Informations- und Datenmengen komplexer. Das kann dazu führen, dass die ursprünglich festgelegten Ziele im Laufe von Projekten schwerer nachzuhalten sind. In der Folge können Kommunikationskonflikte, intransparente Leistungsüberschneidungen und aufwendige Zuarbeiten entstehen. Das trifft auch auf Projekte im Wasserbau zu, die sich durch eine hohe Anzahl an Stakeholdern auszeichnen. Die Harmonisierung der Ansprüche kann zu langwierigen Planungs- und Genehmigungsprozessen führen. Diese Problematik wird in dem vorgestellten Forschungsvorhaben untersucht, um zu prüfen, ob durch Einsatz der künstlichen Intelligenz (KI) eine neue Form der interessengruppenorientierten Prozesssteuerung entstehen kann.

Stichworte: Künstliche Intelligenz, Data Mining, intelligente Dokumentationsassistenten, interessengruppenorientierte Prozesssteuerung
\end{abstract}

\section{Einleitung}

\subsection{Wertschöpfung durch künstliche Intelligenz}

Die fortschreitende und stetige Digitalisierung der Gesellschaft mit den damit verbundenen Veränderungen ist heute auch im Alltag der Unternehmen angekommen. Technologien wie Cloud Computing, die zunehmende Automatisierung, z. B. im Sinne von Industry 4.0-Ansätzen, oder der Einsatz mobiler Endgeräte sind nur einige Beispiele für die Digitalisierung, die den Unternehmen ganz neue Möglichkeiten im Un- 
ternehmensalltag eröffnen. Diese neuen technologischen Möglichkeiten in Kombination mit der weiteren Integration intelligenter Objekte, die die physische mit der digitalen Welt verschmelzen, führen zu neuen und grundlegenden Paradigmenwechseln. Insgesamt verschwimmen durch diese zunehmenden Digitalisierungsmöglichkeiten für die Unternehmen die Grenzen der wertschöpfenden und unterstützenden Prozesse, wodurch sich die traditionellen Lieferketten- und StakeholderBeziehungen eines Unternehmens mit ihren nachgelagerten Prozessen zu einem ganzheitlichen Wertschöpfungsnetzwerk entwickeln. Diese Entwicklungen haben daher entscheidende technologische und vielfältige organisatorische Auswirkungen, wie z.B. drastische Veränderungen der Geschäftsprozesse, Umsetzung neuer Geschäftsmodelle, erhöhte Anforderungen an das Business Process Reengineering, etc. Diese verstärkte Vernetzung von Prozessen und Informationssystemen und damit die zunehmende Menge an Daten, die gemeinsam genutzt, übertragen, gespeichert und analysiert werden müssen, wird von Unternehmen jedoch häufig als Problem wahrgenommen (Bley, Leyh \& Schäffer 2016; Leyh et al. 2017; Mathrani, Mathrani \& Viehland 2013; Pagani 2013).

Aufgrund der Vielfältigkeit der Softwarelandschaft eines Unternehmens selbst aber auch im gesamten Wertschöpfungsnetzwerk in Kombination mit der stetig wachsenden Vernetzung der unterschiedlichen Softwaresysteme werden die Daten selbst zu einem wesentlichen und kritischen Faktor. Daten werden auch weiterhin die Grundlage der digitalen Wirtschaft sein und mehr noch als im letzten Jahrzehnt müssen Unternehmen heute, um im globalen Geschäftsumfeld wettbewerbsfähig zu werden und zu bleiben, große Datenmengen automatisiert zwischen internen Abteilungen und über Unternehmensgrenzen hinweg mit Geschäftspartnern austauschen (Peppard \& Rylander 2006; Sambamurthy, Bharadwaj \& Grover 2003). Neben den technischen Aspekten der Datenerfassung, des Datenaustauschs und der Datenverteilung gewinnen sozioökonomische Fragen zur Wertigkeit und Bewertung von Daten, deren konkreter Nutzung in unterschiedlichen Anwendungskontexten und die zukünftige Organisation von Wertschöpfungsnetzwerken zunehmend an Bedeutung. So ist es nicht verwunderlich, dass heutzutage Themen wie Datenmanagement, Datenanalyse, Künstliche Intelligenz oder Data Science für Unternehmen zunehmend an Bedeutung gewinnen. Immer mehr Unternehmen erkennen das Potenzial von Daten und deren Auswertemethoden. Allerdings sehen sich Unternehmen mit zahlreichen Herausforderungen konfrontiert, wie z. B. unzureichende Datenqualität, Einschränkungen der eigenen Datenarchitektur oder des Datenschutzes (siehe z. B.: Österle \& Otto 2014) oder 
fehlendes Wissen, welches konkrete Werkzeug, welcher Algorithmus, welche Methode ihnen helfen könnte, den Nutzen und Wert ihrer Daten zu steigern.

\subsection{Untersuchung einer KI-gestützten Wertschöpfung der Daten im Be-} reich des Wasserbaus auf einem realen Praxisbeispiel

Aufgrund des hohen Komplexitätsgrads der meisten Wasserbauprojekte, hat das Unternehmen seecon Ingenieure $\mathrm{GmbH}$ sich entschieden, das laufende Vorhaben "Los2: Zwenkau" auszuwählen, um schneller zu marktrelevanten, praxisnahen Erkenntnissen während der Forschungslaufzeit zu kommen. Im Rahmen des Gemeinschaftsprojekts "Data Mining und Wertschöpfung" wird die seecon Ingenieure GmbH zusammen mit dem Fraunhofer-Zentrums für Internationales Management und Wissensökonomie (IMW) und der Fakultät für Informatik der Universität Leipzig eine neue Art der interdisziplinären Projektkommunikation und Durchführung von Bauvorhaben erarbeiten

Auf der Basis von Künstlicher Intelligenz werden strukturierte Datencontainer, intelligente Dokumentationsassistenten (Intelligent Filing Service) und vernetzte Nutzer*innenprofile eingesetzt. Das Ziel ist, eine neue HOAI-basierte Kooperationsbasis zu schaffen, die sich den steigenden Herausforderungen und Möglichkeiten der Digitalisierung in der Baubranche stellen kann.

\subsection{Methodologie}

An die Forschungsarbeit wird polymethodisch herangegangen. Das Quellen untersuchende Vorgehen beinhaltet Literaturrecherchen hinsichtlich Wertschöpfungskonzepten von Daten (u. a. Österle, Otto; Pagani), Business Intelligence (u. a. Hambuch), Datencontainer, Metadatenmanagement und Graphen (u. a Auch; Zane). Darüber hinaus werden die Sekundärquellen zum Pilotprojekt "Los 2 Zwenkau" recherchiert (v. a. Planungsdokumentation). Empirisch sowie explorativ werden diverse Case Studies analysiert sowie Workshops durchgeführt, in der Messinstrumente zur Stakeholder- und Prozessanalyse, wie z. B. morphologischer Kasten, angewendet werden. Dies wird die Basis für das anwendungsausgerichtete Vorgehen sein, das zur Schöpfung eines Mashine Learning Algorithmus führen soll.

\subsection{Ziel des Forschungsvorhabens}

In erster Linie soll ein Wertschöpfungskonzept entstehen, das durch den Einsatz eines selbstlernenden Systems die Zusammenhänge, Aufgaben und Verantwortungsspielräume der jeweiligen Planungsakteure 
aufgreift. Darüber hinaus sollen auf diese Weise die auf einen Planungsserver hochgeladenen Dateien durch ein Intelligent Filing System (IFS) automatisch den entsprechenden Stakeholdern zugewiesen werden. In der zweiten Linie wird ein Algorithmus, der dies ermöglich auf einer Open-Source-Lizenz der Öffentlichkeit zur Verfügung gestellt.

Kurzfristig wird der wertschöpfende Ansatz durch eine deutliche Zeitersparnis vieler Planungsprozesse gewährleistet. Langfristig soll das Ergebnis des Gemeinschaftsprojekts den Ingenieursbüros dabei helfen, sich effizienter und schneller auf die Digitalisierungswende vorzubereiten. Es soll v. a. das Knowhow zur Entwicklung und Benutzung von digitalen Produkten bringen sowie effektiv die Innovation zum Business Development fördern.

\section{2 "Los 2: Wiederherstellung des Vorflutsystems südlich des ehemaligen Tagebaus Zwenkau"}

\subsection{Projektbeschreibung}

Im Braunkohlegebiet Zwenkau-Cospuden sind Möglichkeiten zur Herstellung eines sich weitgehend selbst regulierenden Gebietswasserhaushaltes unter Berücksichtigung wasserwirtschaftlicher, ökologischer, regionalplanerischer und wirtschaftlicher Belange sowie bestehender und geplanter Nutzungen herauszuarbeiten, deren Machbarkeit, Nachhaltigkeit und Einklang mit der Europäischen Wasserrahmenrichtlinie zu prüfen und ihre sonstigen Auswirkungen (Vorteile und Nachteile) aufzuzeigen.

Es wird gefordert, die bergbaubedingt gekappten Wasserläufe zu reaktivieren. Hierzu sind eine Reihe von Teilmaßnahmen, die sich unterteilen lassen in Maßnahmen des Wasserbaus, Ingenieurbaus und der Verkehrsanlagen, notwendig.

Zu den zu bearbeitenden Gewässern gehört die ehemalige Regulierte Weiße Elster mit dem Elsterwehr, die Batschke, der Profener Elstermühlgraben, diverse Grabensysteme sowie der Altarm der Weißen Elster (Alte Elster). Hinzu kommen verschiedene Abschlag-, Zulauf-, Sperr- und Brückenbauwerke sowie Zuwegungen.

\subsection{Planungsinhalt, Leistungsspektrum und Planungsakteure (Stakeholder)}

Das Projekt beinhaltet Leistungen für Ingenieurbauwerke, Verkehrsanlagen, Tragwerksplanungen und naturschutzfachliche Gutachten in 
einem Betrachtungsgebiet mit einer Fläche von ca. 875 ha, die zusammen für eine hohe Komplexität der Planungsinhalte und -prozesse sorgen.

Das Vorhaben bündelt bislang 8 Akteure: die LMBV als AG, die ARGE ihc/seecon als Planer, die Sweco GmbH als Projektsteuerer sowie die Stadt Zwenkau, die Landestalsperrenverwaltung Sachsen, die Landesdirektion Sachsen, den Landkreis Leipzig und den Regionalen Planungsverband Leipzig-Westsachsen. Insgesamt werden ca. 30 Personen an dem Projekt im Zeitraum von 2015 bis 2021 beteiligt sein.

\subsection{Kommunikation zwischen Stakeholder, Datensammlung}

Das Projekt "Los 2 Zwenkau" wird auf klassische Weise gesteuert. Die Hauptkommunikation sowie der Informationsaustausch werden durch Beratungen von Auftraggeber und Planer-ARGE, Protokollierung, diverse Besprechungen sowie Versenden von E-Mails und Durchführung von Telefonaten realisiert. Die Abspeicherung des E-Mailverkehrs sowie neuer Planungsstände sowie außerhalb von Beratungen getroffener Entscheidungen ist notwendig, um Planungsziele zeitlich und wirtschaftlich einzuhalten sowie Nachträge und Kommunikationsprobleme zu vermeiden. Da es kein nutzerorientiertes, automatisches Zuweisungssystem gibt, benötigen diese Aufgaben viel Zeit und Achtsamkeit.

Der Datenaustausch erfolgt durch Exportieren und Importieren der im Vorfeld vorbereiteten und mit Indices zugewiesenen Dateien auf den webbasierten Planungsserver. Die Dokumente können nach Ablageort sowie Dateiname (Index) identifiziert werden, sie sind jedoch keinen konkreten Nutzern zugeordnet, sondern den generellen Nutzergruppen gemäß ihrer Rolle im Projekt. Die Stakeholder erhalten eine E-MailBenachrichtigung über neue Dateien, deren Verwendung auf die Eigennutzung begrenzt ist (z. B. es gibt kein Koordinationsmodell in der Cloud).

\section{Intelligente Datencontainer durch maschinelles Lernen und Datenstrukturen}

Das Datenmanagement und die Verwaltung von Daten erfolgt in modernen Systemen über die Auswertung von Metadaten, die an die Dateien angehängt werden (Auth, 2004; Hambuch, 2008). Die Informationen reichen von Zeitstempeln der Erstellung oder Autoren bis hin zu 
Geokoordinaten von Bildaufnahmen. Die Voraussetzung für die Anwendung dieser Methode ist die Existenz von Metadaten in den Daten. Planende Ingenieure befinden sich hier in einer unkomfortablen Situation, da viele der Daten, die innerhalb der Projektplanung benötigt werden, von weiteren Dienstleistern erstellt und verfügbar gemacht werden müssen. Die Verfügbarkeit von Metadaten entzieht sich aber der eigenen Kontrolle und Qualitätssicherung. Andere Daten werden aus Archiven bereitgestellt und werden teilweise nachdigitalisiert. Die Verfügbarkeit von Metadaten ist gescannten Dokumenten ist kritisch. Hier wird deutlich, dass die automatische Erstellung von Metadaten für die Organisation von Datenmengen ein enormes Potential bietet. Wenn es gelingt zentrale Informationen aus den Daten selbst zu erstellen, so wäre deren Verwaltung einfacher und unabhängig vom Erstellungsprozess der Daten.

Ein Baustein für die Automatisierung der Erstellung von Metadaten ist die Datenanalyse mit Methoden des maschinellen Lernens. Eine grundlegende Anwendung ist die Erkennung von Text in gescannten Dokumenten. Mit Hilfe der Optical Character Recognition (OCR) werden Textbestanteile erkannt und maschinenlesbar. Die erkannten Texte sind zunächst Textartefakte und haben für die Datenrepräsentation keine Bedeutung. Mit Methoden der automatischen Sprachverarbeitung können diesen Artefakten allerdings Bedeutungen zugeordnet werden. So können Adressköpfe erkannt werden, Briefinhalte erfasst werden oder Konzepte wie Gewerke und Dokumenttypen zugeordnet werden. Diese extrahierten Informationen, sozusagen die Umwandlung unstrukturierter Inhalte in strukturierte Informationen, diesen als Fingerabdruck der Dokumente und ersetzen die manuell erzeugten Metadaten. So verfügbare Informationen können in der Datenverwaltung genutzt werden, um die Dokumente und Datenbestände von Bauprojekten auch zu vernetzen. Beispielsweise werden Dokumente von Personen oder Unternehmen zur Verfügung gestellt. Sie beziehen sich auf bestimmte Bauabschnitte. Teile der Dokumente sind als Gutachten identifizierbar, andere wiederum stellen Rechnungen dar. Die Verbindungen zu Personen, Gewerken, Bauabschnitten oder gar Projektphasen können in einer intelligenten Datenstruktur aufgehen, welche die nötigen Verbindungen zwischen den beteiligten Stakeholdern und den Projektdaten herstellt. Die genannten Verbindungen können über Graphdatenbanken realisiert werden, deren Kernkompetenz die Repräsentation von Daten und deren Verknüpfungen darstellt (Eifrem, 2015; Zane, 2016). 
Ein Graph ist eine Menge von sogenannten Knoten, welche die Daten, wie zum Beispiel Dokumente, darstellen. Diese Knoten werden untereinander mit sogenannten Kanten verbunden, um die Daten miteinander zu verknüpfen. Zusätzlich können die Kanten auch Informationen speichern, um die Art der Verknüpfung zu speichern. Durch die Art der Speicherung der Daten sind Informationen in verknüpfter Form verfügbar und repräsentieren so explizite und implizite Informationen. Verknüpfte Informationen erlauben einen effizienten Zugriff auf in den Daten enthaltenes Wissen. Durch die Projektion dieser Verknüpfungen in räumliche Darstellungen, wie zum Beispiel Karten oder Gebäudemodelle, können zudem auch spatiale Zusammenhänge aufgedeckt werden. Hierbei können zum Beispiel Gebiete mit ähnlichen Beschreibungen in den Gutachten miteinander verknüpft werden.

Die Verknüpfung der Daten wird durch einen KI-gestützten Workflow erstellt und bietet dann dem planenden Ingenieur ein datengestütztes Assistenzsystem. Das System unterstützt die tägliche Arbeit der Planung, das Datenmanagement tritt in den Hintergrund und die Kernaufgaben des planenden Ingenieurs treten wieder in dem Vordergrund.

\section{Fazit und Diskussion}

Die frühzeitige Identifizierung der Marktveränderungen und eine Anpassungsfähigkeit der Planungs- und Ingenieurbüros sind in Zeiten der digitalen Ära unverzichtbar. Die stets wachsenden Anforderungen an Innovation, Schnelligkeit und intersektorale Kooperation transformieren das Profil des sozio-ökonomische Nachfrage-Bedarf Verhältnisses und führen zu konsequenten Umstrukturierungen des Dienstleistungsspektrums innerhalb der Ingenieurbranche. Die neuen Geschäftsmodelle und daran angeknüpften Wertschöpfungskonzepte, die Daten als Rohstoff betrachten, sind einer der Möglichkeiten, sich besser in einem Markt zu positionieren sowie schneller an Bedürfnisse des Kunden zu reagieren.

Durch die Automatisierung sich wiederholender Aufgaben, wie Zuordnung der Daten oder Zuweisung der Daten und Aufgaben an die durch festgestellten Planakteure mit IFS, wird der erste Schritt zur Deckung der immer geringeren Zeiträume für Fertigstellung einer Leistung abgesichert. Im Laufe der Forschungsarbeit sollen mehrere kritische Stellen identifiziert werden, die eine ähnliche Zeitersparnis ermöglichen. Ebenso wichtig ist es herauszufinden, in welcher Form der Algorithmus in die Planungsinfrastruktur implementiert werden soll bzw. welche 
Kompetenzen und IT-Infrastruktur solch eine Implementierung erfordert.

Der tatsächliche Mehrwert besteht darin, Unternehmen bei der Anpassung an die digitale Transformation sowie bei dem Umgang mit Innovation zu helfen.

\section{$5 \quad$ Literatur}

Auth, G. (2004). Prozessorientierte Organisation des Metadatenmanagements für Data-Warehouse-Systeme: Mit 80 Tabellen. Books on Demand GmbH.

Bley, K., Leyh, C. \& Schäffer, T. (2016): Digitization of german enterprises in the production sector - do they know how "digitized" they are? In: Proceedings of the Americas Conferenence on Information Systems, AMCIS 2016, 11-14 August, San Diego, USA 2016.

Eifrem, I. Robinson. J. Webber. E. (2015). Graph Databases, 2nd Edition. O'Reilly Media, Inc. http://proquest.safaribooksonline.com/9781491930885

Leyh, C., Schäffer, T., Bley, K. \& Forstenhäusler, S. (2017): Assessing the IT and Software Landscapes of Industry 4.0-Enterprises: The Maturity Model SIMMI 4.0. In Information Technology for Management: New Ideas and Real Solutions, E. Ziemba (Hrsg.), Springer, Cham 2017, S. 103-119.

Hambuch, U. (2008). Erfolgsfaktor Metadatenmanagement: Relevanz des Metadatenmanagements für die Datenqualität bei Business Intelligence. VDM Verl. Dr. Müller.

Mathrani, S., Mathrani, A. \& Viehland, D. (2013): Using enterprise systems to realize digital business strategies. In: Journal of Enterprise Information Management, Vol. 26, No.4, 2013, S. 363- 386.

Österle, H. \& Otto, B. (2014): Das datenzentrierte Unternehmen: Eine Business-Engineering-Perspektive. In: Enterprise-Integration, G. Schuh \& V. Stich (Hrsg.), Springer Vieweg, Heidelberg 2014, S. 91 105.

Pagani, M. (2013): Digital Business Strategy and Value Creation: Framing the Dynamic Cycle of Control Points. In: MIS Quarterly, Vol. 37, No. 2, 2013, S. 617-632.

Peppard, J. \& Rylander, A. (2006): From value chain to value network: insights for mobile operators. In: European Management Journal, Vol. 24, No. 2-3, 2006, S. 128-141.

Sambamurthy, V., Bharadwaj, A. \& Grover, V. (2003): Shaping agility through digital options: reconceptualizing the role of information technology in contemporary firms. In: MIS Quarterly, Vol. 27, No. 2, 2003, S. 237-263.

Zane, B. (2016, November 2). Semantic Graph Databases: A worthy successor to relational databases. Database Trends and 
Applications.

http://www.dbta.com/BigDataQuarterly/Articles/Semantic-GraphDatabases-A-worthy-successor-to-relational-databases114569.aspx

Autoren:

Patrycja-Jadwiga Sankowska

seecon Ingenieure $\mathrm{GmbH}$

Stabstelle Digitalisierung

Spinnereistr. 7, Halle 14

04179 Leipzig

$\begin{array}{ll}\text { Tel.: } & +493414840528 \\ \text { Fax: } & +493414840520 \\ \text { E-Mail: } \quad \text { patrycja.sankow- } & \text { ska@seecon.de } \\ \text { sk. Christian Leyh }\end{array}$

\author{
Nina Kumbruck \\ seecon Ingenieure $\mathrm{GmbH}$ \\ Fachbereich Wasserbau \\ Spinnereistr. 7, Halle 14 \\ 04179 Leipzig
}

$\begin{array}{ll}\text { Tel.: } & +493414840530 \\ \text { Fax: } & +493414840520 \\ \text { E-Mail: } & \text { nina.kumbruck@se- } \\ \text { econ.de } & \end{array}$

Dr. Andreas Niekler

Dr. Daniel Wiegreffe
Fraunhofer-Zentrum für internationales Management und Wissensökonomie IMW

Digitale Projekteinheit Data Mining und Wertschöpfung

Neumarkt 9-19

04109 Leipzig

Tel: +49341231039279

E-Mail: christian.leyh@imw.fraunhofer.de
Universität Leipzig

Projekt Data Mining und Wertschöpfung, Institut für Informatik

Augustusplatz 10

04109 Leipzig

Tel: +49341231039279

E-Mail: aniekler,daniel@informatik.uni-leipzig.de 